\title{
Public health aspects of obstructive sleep apnoea
}

\author{
G J Gibson
}

The following three articles in this issue of Thorax $^{1-3}$ summarise the contributions to a lively symposium on public health aspects of obstructive sleep apnoea (OSA) which I had the pleasure of chairing at the meeting of the British Thoracic Society in December 1997. The symposium followed the publication in the $B M \mathcal{F}$ of a systematic review by Wright and colleagues on the health effects of obstructive sleep apnoea and the effectiveness of continuous positive airway pressure. ${ }^{4}$ Their main conclusions were that the relevance of sleep apnoea to public health had been exaggerated and the effectiveness of treatment with continuous positive airway pressure (CPAP) had been poorly evaluated. The authors questioned whether sleep apnoea was "a separate disease entity or a marker or a symptom of obesity and ageing" and they suggested that the morbidity associated with sleep apnoea was due to confounding factors, particularly obesity. In relation to CPAP treatment, they concluded that the quality of controlled trials was "poor" and that no studies had included "an adequate placebo". Anecdotal evidence from several centres suggests that the final sentence of their paper: "Calls for widespread investment in health service provision in this topic may be premature" has been taken to heart in considerable measure by purchasing authorities throughout the UK and further afield.

The juxtaposition in the paper by Wright et $a l^{4}$ of reviews of the cardiovascular morbidity and mortality possibly associated with sleep apnoea and the efficacy of treatment with CPAP could easily lead the clinically naive purchaser to infer that CPAP treatment is used to prevent such long term vascular consequences. As Davies ${ }^{2}$ points out, this is not the case - the aim of treatment is to control the morbidity associated with disabling sleepiness. As it happens, a similar review of CPAP treatment was carried out almost simultaneously by the Australian National Health and Medical Research Council. ${ }^{5}$ Wright and Sheldon ${ }^{1}$ correctly point out that nine of the 10 recommendations in the Australian report agreed with their own. The difference, of course, lay in the all important first recommendation in the Australian report that CPAP treatment was indicated for symptomatic patients with OSA.

Some estimates of the potential impact of sleep apnoea and the consequent financial implications have sent shudders down the public health spine and the case of patients with disabling sleep apnoea may not have been helped by such extravagant claims. Nonetheless, it is now abundantly clear that symptomatic OSA is very common and undoubtedly a cause of considerable cumulative morbidity, albeit not necessarily vascular morbidity. Wright and Sheldon ${ }^{1}$ pose important specific questions. Firstly, "what is sleep apnoea and how common is it?", pointing out that immediately we run into problems of changing definitions. A major difficulty in this area is the lack of a robust method of categorising patients with OSA and related conditions. This is less important in sleepy patients with a very high apnoea/hypopnoea index (AHI), but there are also patients with high AHI values and few symptoms (who are likely to be picked up in epidemiological studies) and a larger group within the hospital population with troublesome symptoms and little apparent "abnormality" in terms of AHI. The problems of imprecise definition of disease will not be unfamiliar to respiratory physicians who have struggled for nearly half a century to distinguish between asthma and COPD! Wright and Sheldon ${ }^{1}$ conclude reasonably that the definition should be developed in the context of the impact of treatment. They then address the question: "is there a causal link between sleep apnoea and morbidity and mortality?", defining the criteria which should be used in answering such a question and raising the important role of confounding factors, particularly obesity. There is considerable agreement between them ${ }^{1}$ and Davies ${ }^{2}$ on the unconvincing nature of much of the evidence linking OSA with sustained daytime hypertension, ischaemic heart disease, and stroke. Davies ${ }^{2}$ makes the additional important point that mean nocturnal blood pressure is clearly raised in patients with untreated OSA, a point which should not be dismissed in view of the evidence in essential hypertension that nocturnal blood pressure has an independent adverse prognostic effect. Recent data from the large epidemiological study in Wisconsin ${ }^{6}$ offer the most convincing evidence to date that OSA may indeed have an independent effect on daytime blood pressure, but it seems unlikely to be as large an effect as that associated with other important risk factors such as obesity.

Turning to treatment, Wright and Sheldon ${ }^{1}$ restate their criticisms of the only randomised controlled study of treatment published at the time of their original review. ${ }^{4}$ Not surprisingly, Douglas ${ }^{3}$ offers a detailed and robust defence of this study and he usefully summarises four further studies subsequently published in full or as an abstract, one of which is published in this issue of Thorax. ${ }^{7}$ Wright and Sheldon's main criticism of the Edinburgh studies continues to be the placebo used (a tablet), but they underestimate the difficulty of finding an appropriate placebo for such studies. Unlike conventional randomised control trials of pharmacological treatment, there is no satisfactory placebo for nasal CPAP. Clearly a tablet is less than ideal but, for the reasons expounded by Douglas, ${ }^{3}$ nor is "sham CPAP" perfect. 
Hopefully, the accumulating evidence from the five controlled studies reviewed by Douglas ${ }^{3}$ will satisfy the planners of health care but, in my view, the expectation that further trials of larger numbers of patients will simplify clinical decisions is naive and illusory. Unlike the situation with pharmacological therapy, CPAP treatment is initiated only after a trial period and, even if we can improve recognition of those patients more likely to benefit, the final decision will still depend on a therapeutic trial in each individual.

Among other forms of treatment there is reasonable agreement that uvulopalatopharyngoplasty and similar procedures have a very small role in patients with symptomatic OSA. Intraoral devices offer more hope but, even though patients in the three studies quoted by Wright and Sheldon ${ }^{1}$ preferred such a device to CPAP, it should be noted that they were less effective in reducing AHI and in two of the studies the population was deliberately biased towards milder disease. Wright and Sheldon ${ }^{1}$ point out that the three studies of intraoral appliances included more patients than had been studied in trials of CPAP alone-but it seems entirely appropriate to devote scarce research funds to the study of areas where genuine uncertainty exists, rather than to those where the effects are clearly evident to all who care to look.

Wright and Sheldon ${ }^{1}$ reiterate their earlier statement that "sleep apnoea is a symptom of obesity" and they re-emphasise the importance of achieving weight loss. Clearly, there is an important association between OSA and obesity, but obesity is also associated with hypertension and diabetes and claims that these diseases were merely "symptoms" of obesity would not be taken seriously. Although OSA was first described in the very obese, it is now clear that a large proportion of patients are not overweight. It may be that the mental image of non-clinicians is coloured by outdated pictures of the "Pickwickian syndrome" as representing the typical patient with OSA. For several reasons, effective treatment of obesity is of undoubted importance and if weight loss can be achieved it certainly can produce marked improvement in obese patients with OSA. However, weight reduction is much easier to advocate than to achieve. Generally, it is more effective if tailored to the individual patient ${ }^{8}$ and if more than one type of treatment modality is used simultaneously. At best, however, success is modest and frequently unsustained. ${ }^{9}$ It is also important to remember that patients in studies of weight loss are much more likely to be motivated to lose weight than is the typical somnolent patient with OSA. Treatment of OSA with CPAP is certainly safer than weight reducing pills and it is more immediately effective (and probably much cheaper) than attempts to achieve weight loss.

The review by Wright $e t a l^{4}$ has undoubtedly had a major impact on the sleep apnoea world. The lack of controlled studies of CPAP treatment has been acknowledged and is being met. However, the need to translate the results of trials, particularly in patients with less severe OSA, to individual clinical practice will remain, and patients with symptomatic OSA will continue to require individual trials of treatment. Our contributors are agreed over the lack of robust evidence that OSA causes significant vascular disease or that CPAP treatment influences this. There is also increasing acknowledgement that CPAP treatment helps sleepiness, at least when this is severe. It is encouraging that Wright and Sheldon ${ }^{1}$ share the concerns of clinicians over how some purchasing authorities have interpreted the results of their earlier review, but the more cynical might think that such responses were entirely predictable. They make the important practical point that increased resources for investigation and treatment of sleep apnoea might mean less to treat patients with other forms of respiratory disease. Physicians treating OSA feel that their patients are being "victimised" in that sleep apnoea is relatively newly understood (or misunderstood) and is vulnerable to funding restrictions because of its "newness", apparent lack of urgency, and the absence of advocates among those making financial decisions. The improvements in many patients are dramatic and very rewarding and attention needs to be given to better methods of quantifying and publicising the benefits of treatment.

1 Wright J, Sheldon T. Sleep apnoea and its impact on public health. Thorax 1998;53:410-3.

2 Davies RJO. Cardiovascular aspects of obstructive sleep apnoea and the effect of nasal CPAP. Thorax 1998;53:4168.

3 Douglas NJ. Systematic review of the efficacy of nasal CPAP. Thorax 1998;53:414-5.

4 Wright J, Johns R, Watt I, et al. Health effects of obstructive sleep apnoea and effectiveness of continuous positive airway pressure: a systematic review of the research airway pressure: a systematic
evidence. $B M \mathcal{F}$ 1997;314:851-60.

5 Australian Health Technology Advisory Committee. The effectiveness and cost effectiveness of nasal continuous positive airway pressure in the treatment of obstructive sleep apnoea in adults. Government Public Services, Canberra, 1996.

6 Young T, Peppard P, Palta M, et al. Population-based study of sleep-disordered breathing as a risk factor for hypertension. Arch Intern Med 1997;157:1746-52.

7 Engleman HM, Martin SE, Kingshott RN, et al. Randomised placebo controlled trial of daytime function after continuous positive airway pressure (CPAP) therapy for the sleep apnoea/hypopnoea syndrome. Thorax 1998;53:3415 .

8 Royal College of Physicians. Overweight and obese patients. London: Royal College of Physicians, 1997.

9 NHS Centre for Reviews and Dissemination. The prevention and treatment of obesity. Effective Health Care 1997;3: $1-11$ 